EGU21-9057, updated on 09 May 2021

https://doi.org/10.5194/egusphere-egu21-9057

EGU General Assembly 2021

(c) Author(s) 2021. This work is distributed under

the Creative Commons Attribution 4.0 License.

\title{
Differentiating between primary and secondary organic aerosols of biomass burning in an environmental chamber with FTIR and AMS
}

\author{
Amir Yazdani ${ }^{1}$, Satoshi Takahama ${ }^{1}$, Jack K. Kodros ${ }^{2}$, Marco Paglione ${ }^{2,3}$, Mauro Masiol ${ }^{2}$, Stefania \\ Squizzato ${ }^{2}$, Kalliopi Florou ${ }^{2}$, Spyros N. Pandis ${ }^{2}$, and Athanasios Nenes ${ }^{1,2}$ \\ ${ }^{1}$ ENAC/IIE Swiss Federal Institute of Technology Lausanne (EPFL), Lausanne, Switzerland \\ ${ }^{2}$ Institute for Chemical Engineering Sciences, Foundation for Research and Technology Hellas (ICE-HT/FORTH), Patras, \\ Greece \\ ${ }^{3}$ Italian National Research Council - Institute of Atmospheric Sciences and Climate (CNR-ISAC), Bologna, Italy
}

Fine particulate matter (PM) affects visibility, climate and public health. Organic matter (OM), which is hard to characterize due to its complex chemical composition, can constitute more than half of the PM. Biomass burning from residential wood burning, wildfires, and prescribed burning is a major source of OM with an ever-increasing importance.

Aerosol mass spectrometry (AMS) and Fourier transform infrared spectroscopy (FTIR) are two complementary methods of identifying the chemical composition of OM. AMS measures the bulk composition of OM with relatively high temporal resolution but provides limited parent compound information. FTIR, carried out on samples collected on Teflon filters, provides detailed functional groupinformation at the expense of relatively low temporal resolution.

In this study, we used these two methods to better understand the evolution of biomass burning OM in the atmosphere with aging. For this purpose, primary emissions from wood and pellet stoves were injected into the Center for Studies of Air Qualities and Climate Change (CSTACC) environmental chamber at ICE-HT/FORTH. Primary emissions were aged using hydroxyl and nitrate radicals (with atmospherically relevant exposures) simulating atmospheric day-time and night-time oxidation. A time-of-flight (ToF) AMS reported the composition of non-refractory $\mathrm{PM}_{1}$ every three minutes and $\mathrm{PM}_{1}$ was collected on PTFE filters over 20-minute periods before and after aging for off-line FTIR analysis.

We found that AMS and FTIR measurements agreed well in terms of measured OM mass concentration, the $\mathrm{OM}: \mathrm{OC}$ ratio, and concentration of biomass burning tracers - lignin and levoglucosan. AMS OM concentration was used to estimate chamber wall loss rates which were then used separate the contribution of primary and secondary organic aerosols (POA and SOA) to the aged OM. AMS mass spectra and FTIR spectra of biomass burning SOA and estimates of bulk composition were obtained by this procedure. FTIR and AMS spectra of SOA produced by $\mathrm{OH}$ oxidation of biomass burning volatile organic compounds (VOCs) were dominated by acid signatures. Organonitrates, on the other hand, appeared to be important in the SOA aged by the nitrate radical. The spectra from the two instruments also indicated that the signatures of certain 
compounds such as levoglucosan, lignin and hydrocarbons, which are abundant in biomass burning POA, diminish with aging significantly more than what can be attributed to chamber wall losses. The latter suggests biomass burning POA chemical composition might change noticeably due to heterogeneous reactions or partitioning in the atmosphere. Therefore, the common assumption of stable POA composition is only partially true. In addition, more stable biomass burning tracers should be used to be able to identify highly aged biomass burning aerosols in the atmosphere. 